\title{
Journalismuskultur: Zur Dimensionierung eines zentralen Konstrukts der kulturvergleichenden Journalismusforschung
}

\author{
Thomas Hanitzsch
}

Trotz der zunebmenden Bedeutung von Journalismuskultur als heuristisches Denkwerkzeug vor allem für die komparative und kultursoziologisch motivierte Journalismusforschung hat eine theoretische Auseinandersetzung mit diesem Konzept bislang kaum stattgefunden. Der vorliegende Beitrag versucht diese Lücke zu schließen, indem er ein Modell entwickelt, dessen Abstraktionsgrad und Universalität es erlaubt, die Vielfalt journalistischer Kulturen anhand ibrer zentralen Basiselemente abzubilden und auf einen gemeinsamen theoretischen Nenner zu bringen. Anhand des Forschungsstandes und der zugänglichen Literatur wird vorgeschlagen, Journalismuskultur in seine dimensionale Struktur zu dekonstruieren. Demnach setzt sich Journalismuskultur aus drei Konstituenten zusammen (institutionelle Rollen, Epistemologien und ethische Ideologien), die wiederum in sieben kontinuierliche Hauptdimensionen zerlegt werden können: Interventionismus, Machtdistanz, Marktorientierung, Objektivismus, Empirismus, Relativismus und Idealismus. Damit eröffnet das Modell einen siebendimensionalen Raum, in dem verschiedene journalistische Kulturen systematisch verglichen und taxonomisch eingeordnet werden können.

Schlagwörter: Journalismuskultur, komparative Journalismusforschung, berufliches Selbstverständnis, Berufsethik, journalistische Epistemologien

\section{Zur Einführung}

Mit Journalismuskultur gewinnt in letzter Zeit ein Konzept an Schärfe, das die komparative Journalismusforschung insbesondere seit den 1990er Jahren latent inspiriert hat. Gemeint war die - mit dem Blick auf den Forschungsstand konstatierte - Ambivalenz von zunehmenden Ähnlichkeiten bei gleichzeitig immer noch teilweise gravierenden Unterschieden im Hinblick auf die Merkmale und professionellen Orientierungen von Journalisten (vgl. Deuze 2002; Donsbach \& Patterson 2003; Esser 1998; Scholl \& Weischenberg 1998; Shoemaker \& Cohen 2006; Splichal \& Sparks 1994; Weaver 1998). Bei dem Versuch, die Pluralität beruflicher Rollenverständnisse und Praktiken in theoretische Konzepte $\mathrm{zu}$ fassen, sprechen Forscher zunehmend von „journalistischen Kulturen“ (vgl. u. a. Campbell 2004; Hanitzsch 2006; Harrison 2000; Hollifield, Kosicki \& Becker 2001; Keeble 2005; Kopper 2003; Machill 1997; Zelizer 2005). Synonym verwendet werden häufig auch Begriffe wie „Nachrichtenkulturen” (vgl. Deuze 2002; Donsbach \& Patterson 2003), „Zeitungskulturen” (vgl. Knott, Carroll \& Meyer 2002) oder „professionelle Kulturen” (vgl. Esser 2004; Fawcett 2002; Loo 1996). Die perspektivische Bandbreite erstreckt sich dabei von einer newsroom ethnography in der britischen Tradition (vgl. Harrison 2000) bis hin zu politikwissenschaftlich inspirierten Konzepten, die in der Nähe von Pfetschs (2003) politischen Kommunikationskulturen anzusiedeln sind.

Trotz der Zentralität des Journalismuskultur-Begriffs für die komparative Journalismusforschung sind bislang nur wenige Versuche unternommen worden, das Konzept 
theoretisch zu durchdringen (vgl. Deuze 2002; Harrison 2000; Kopper 2003). Damit sind einige grundsätzliche Fragen unbeantwortet geblieben: Was genau meint „Journalismuskultur“? Sprechen wir von einer - universellen - Journalismuskultur, oder verweist das Konzept auf das Vorhandensein distinkter journalistischer Kulturen? Auf welcher Analyseebene ist das Konzept anzusiedeln? Wie können unterschiedliche Journalismuskulturen systematisch beschrieben und klassifiziert werden? Was sind die grundlegenden Determinanten von Journalismuskultur? Und: Wie lässt sich Journalismuskultur der empirischen Forschung zugänglich machen?

Die bislang vorliegenden Arbeiten lassen sich überwiegend in das Umfeld der Erforschung professioneller Orientierungen von Journalisten in der Tradition von Köcher (1986), Donsbach und Patterson (2003), Splichal und Sparks (1994) sowie Weaver (1998) einordnen. Dabei zeigt sich ein weiteres Problem: Die Forschung wird von Modellen bestimmt, die in westlichen Kontexten entwickelt wurden und die das globale Spektrum von Journalismuskulturen nur bedingt abbilden können. Viele Studien stützen ihre Untersuchungen auf das Professionalsierungsmodell, dessen normative Erwartungen nicht nur in die Operationalisierung eingehen, sondern möglicherweise auch die Antworten beeinflussen (vgl. Josephi 2006: 584). Andererseits haben Arbeiten, die sich z. B. auf die Suche nach einem „Asiatischen Journalismus“ gemacht haben, allenfalls ambivalente Befunde produziert (vgl. Massey \& Chang 2002; Masterton 1996; Xiaoge 2005).

Damit ist ein zentrales Desiderat der kulturvergleichenden Journalismusforschung umrissen: Es fehlt an einer theoretischen Fundierung des Konzepts Journalismuskultur, die abstrakt und universell genug ist, um die kulturelle Vielfalt journalistischer Orientierungen und Praktiken systematisch und hinreichend abzubilden. Am Ende des Denkprozesses muss daher ein Modell stehen, das die zentralen Basiselemente der Varianz von journalistischen Kulturen aufgreift und das in sehr unterschiedlichen kulturellen Kontexten eingesetzt werden kann. In diese Lücke will der vorliegende Beitrag stoßen, indem er versucht, Journalismuskultur als forschungsleitendes Konstrukt der komparativen Forschung zugänglich zu machen.

\section{Begriffliche Annäherung}

\subsection{Zum Kulturbegriff}

Aufgrund seiner begrifflichen Unschärfe und vielfältigen Verwendung gilt „Kultur“ im aktuellen Diskurs als „ein chronisch vieldeutiges“ (Reckwitz 1997: 319), „gnadenlos inflationäres“ (Karmasin 2000: 245) „Amöbenwort“ (Kramer 2000: 62). Die verwendeten Definitionen reichen dabei von einer Eingrenzung auf Nationalkulturen (vgl. Hofstede 1980) hin zu empirisch schwer fassbaren Einordnungen von Kultur als „Gesamtheit der Lebensweise“ (Williams 1958: 18). Die britische Soziologin Margaret Archer (1996: 2) bedauert daher, dass sich der Kulturbegriff offenbar in einem „konzeptuellen Morast festgefahren“ hat. Auch für die Journalismusforschung tut sich mit der verstärkten Hinwendung zur Kulturanalyse ein disparates Forschungsfeld auf, dessen Grenzen vermutlich erst noch abgesteckt werden müssen.

Wie alle Kulturen können sich auch journalistische Kulturen prinzipiell in Form von (a) Orientierungen (Werte, Einstellungen, Überzeugungen), (b) Praktiken (kultureller 
Produktion) und (c) Artefakten (Produkte, Texte) manifestieren. ${ }^{1}$ Zugang verschafft sich die wissenschaftliche Analyse demnach idealerweise durch (a) die Befragung von Journalisten, (b) die Beobachtung journalistischer Tätigkeit und (c) die Inhaltsanalyse von Berichterstattung. Bei der Analyse kultureller Prozesse - und Journalismus ist ein solcher Prozess (vgl. Lünenborg 2005) - interessieren vor allem professionelle Orientierungen und Praktiken. In einer vorläufigen Annäherung wollen wir Journalismuskultur daher definieren als die im Journalismus spezifischen kognitiven, evaluativen und performativen kulturellen Ordnungen, mit denen sich Journalisten ibre Wirklichkeit als bedeutungsvoll erschaffen und die in Form von kollektiven Wissensordnungen ibr Handeln ermöglichen und einschränken. Im Journalismus finden diese symbolischen Ordnungen ihren Niederschlag auf drei Ebenen:

- Die kognitiven Schemata steuern als Grundelemente der Wahrnehmung und Deutung die routinisierte Nachrichtenproduktion, z. B. die journalistische Relevanzzuweisung an Ereignisse in Form von Nachrichtenwerten.

- Die evaluativen Schemata leiten die in beruflichen Zusammenhängen vorgenommenen Bewertungen an. Dies geschieht in Form von professionellen Weltsichten der Akteure (Rollenverständnis) und professionellen Subkulturen (z. B. „objektiver Journalismus“, „investigativer Journalismus“).

- Die performativen Schemata (z. B. Recherchemethoden) manifestieren sich in der journalistischen Praxis. Die Handlungen der Journalisten werden dabei angeleitet durch kognitive und evaluative Schemata, denen der Praxisvollzug wiederum Geltung verschafft.

Im Folgenden wollen wir uns auf die evaluativen Schemata beschränken, da sie Auskunft über professionelle Selbstbeschreibungen und Identitäten im Journalismus geben. Es ist anzunehmen, dass diese Orientierungen - das legen Forschungen zur Handlungsrelevanz beruflicher Rollenverständnisse nahe - in die journalistische Praxis Eingang finden (vgl. Scholl \& Weischenberg 1998; Shoemaker \& Reese 1996; Weischenberg, Bassewitz \& Scholl 1989).

\section{2 Ähnlichkeit und Differenz}

In der hier vertretenen Lesart verweist das Konzept Journalismuskultur sowohl auf Ähnlichkeit als auch auf Differenz. Ähnlichkeiten manifestieren sich in Form eines ideologischen Konsenses, der von allen Journalisten kollektiv geteilt wird. Die idealtypischen Werte einer solchen universell-„kosmopolitischen“ (Reese 2001: 178) Journalismuskultur können auf sechs Elemente reduziert werden: Journalismus ist (1) eine berufliche Tätigkeit, die sich in (2) organisationalen Strukturen vollzieht. Journalismus ist (3) ein „Dienst an der Gesellschaft“ bzw. am Publikum, stellt (4) aktuelle und relevante Informationen bereit, orientiert sich (5) hauptsächlich an Fakten und erfordert (6) ein Mindestmaß an intellektueller Unabhängigkeit und Autonomie (vgl. u. a. Deuze 2005; Kovach \& Rosenstiel 2001). Diese sechs Elemente bilden das kulturübergreifende Fundament gemeinsam geteilter Werte, auf dem unterschiedliche journalistische Subkulturen ihre Differenz artikulieren.

Die Differenzen zwischen journalistischen Subkulturen manifestieren sich wieder-

1 Auf eine ausführliche Diskussion der verschiedenen perspektivischen Zugänge zur Kulturanalyse muss hier aus Platzgründen verzichtet werden. Dem hier vorgestellten Ansatz unterliegt ein deskriptives Kulturverständnis, d. h., es geht nicht darum, bestimmte Journalismuskulturen aus einer normativen Perspektive kritisch zu hinterfragen. 
um auf drei Aggregatebenen - der Makro-Ebene der nationalen Journalismuskulturen, der Meso-Ebene der journalistischen Organisationskulturen und der Mikro-Ebene der professionellen Milieus:

- Nationale Journalismuskulturen orientieren sich an territorialstaatlichen Grenzen (vgl. Blumler 1983; Donsbach \& Patterson 2003; Weaver 1998). So bezeichnet etwa Donsbach (1995: 25) den deutschen und US-amerikanischen Journalismus als „Zwei sehr verschiedene professionelle Welten“.

- Journalistische Organisationskulturen beschreiben die kollektiven Orientierungen und Praktiken, die Mitglieder einer Organisation von Mitgliedern anderer Organisationen unterscheiden (vgl. Hofstede 2001: 391). Gade (2004: 42ff.) spricht z. B. von einem „Kulturkampf“ $z$ wischen einer redaktionsspezifischen journalistischen Kultur und einer journalismusfremden „Marketingkultur“ in integrierten Redaktionen.

- Professionelle Milieus verbinden Journalisten mit ähnlichen professionellen Orientierungen und Praktiken. Beispiele hierfür sind die Milieus des „Public/civic journalism“ und des „New journalism“, die sich beide gegen den Mainstream eines „objektiv-neutralen Journalismus“ artikulieren.

\subsection{Dimensionale Dekonstruktion}

Für die Analyse verschiedener Journalismuskulturen stellen komparative Forschungsdesigns einen Königsweg dar. Ein zentrales Kriterium ist hierbei die Äquivalenz der forschungsleitenden Konstrukte (Van de Vijver \& Leung 1997: 8), d. h., Journalismuskultur als Denkfigur und zentrales Konstrukt muss sich in verschiedenen kulturellen Kontexten als valides Forschungswerkzeug beweisen. Prinzipiell bieten sich zwei Wege an, um zu einer operationalen Definition der forschungsleitenden Konstrukte zu kommen. Diese können in Anlehnung an eine von Pike (1967) getroffene Unterscheidung als emische und etische Strategien charakterisiert werden:

Der emische Ansatz stellt das Besondere, das Spezifische einer Kultur in den Vordergrund. Kulturelle Zusammenhänge werden mit den Augen eines Insiders betrachtet, womit ein Sinnverstehen von Bedeutungsinhalten ermöglicht wird, die Außenstehenden oft unzugänglich sind. Demgegenüber werden beim etischen Ansatz, dessen Fokus eher auf dem Universellen und Kulturübergreifenden liegt, die konstitutiven Elemente einer Kultur aus der Perspektive eines externen Beobachters erforscht. Durch die Verwendung von abstrakten Konstrukten werden unterschiedliche kulturelle Ausprägungen auf einen gemeinsamen theoretischen Nenner gebracht.

Eine emische Strategie der Identifikation und Beschreibung von Journalismuskulturen würde die forschungsleitenden Konstrukte in einem bestimmten kulturellen Kontext entwickeln. Die auf diese Weise gewonnenen Definitionen sind folglich in erster Linie kulturgebunden, und eine Übertragbarkeit auf andere kulturelle Kontexte wäre in den meisten Fällen kaum gewährleistet. Beispiele hierfür sind die national zum Teil sehr unterschiedlichen Definitionen von "Journalismus" und "Journalist“. Außerdem kann die Auslegung der Funktionalität von Journalismus (z. B. neutrale Beobachtung vs. politische Teilhabe) selbst innerhalb eines Landes stark variieren. Für die komparative Erforschung von Journalismuskulturen erscheint eine etische Vorgehensweise daher am sinnvollsten. ${ }^{2}$ Eine solche deduktive Strategie verspricht nicht nur ein hohes Maß an

2 Emisch motivierte Analysen sollten jedoch einer etischen Strategie unbedingt vorausgehen, denn nur so kann der Forscher Kandidaten für universell gültige Kulturdimensionen generieren. 
Konstruktäquivalenz, sie würde darüber hinaus Definitionen hervorbringen, die dem steten Wandel von Journalismuskulturen Rechnung tragen können.

Mittels einer solchen etisch-deduktiven Vorgehensweise soll Journalismuskultur im Folgenden in seine konstitutiven Elemente dekonstruiert werden. Da es unmöglich ist, alle denkbaren Facetten von Journalismuskulturen in einem Modell abzubilden, war es nötig, eine Auswahl zu treffen. Diese basierte auf der Annahme, dass die ausgewählten Hauptdimensionen die Differenzen zwischen journalistischen Subkulturen hinreichend abbilden und die unvermeidbare Restvarianz gering bleibt. Ein weiteres Selektionskriterium war ihre Prominenz in der wissenschaftlichen Literatur und öffentlichen Debatte. Die Sichtung des Forschungsstandes legt eine Struktur nahe, die aus drei Konstituenten (institutionelle Rollen, Epistemologien und ethische Ideologien) besteht, die wiederum in sieben Hauptdimensionen zerlegt werden können: Interventionismus, Machtdistanz, Marktorientierung, Objektivismus, Empirismus, Relativismus und Idealismus (vgl. Abb. 1).

\section{Abbildung 1: Konstituenten und Hauptdimensionen von Journalismuskultur}

\begin{tabular}{|c|c|c|c|c|}
\hline \multirow{3}{*}{ Institutionelle Rollen } & \multicolumn{2}{|c|}{ Interventionismus: $(+)$ Intervention } & $\leftrightarrow$ & Passiv (-) \\
\hline & Machtdistanz: & (+) Gegenpol & $\leftrightarrow$ & Loyal (-) \\
\hline & Marktorientier & $(+)$ Konsument & $\leftrightarrow \mathrm{St}$ & atsbürger (-) \\
\hline \multirow{2}{*}{ Epistemologien } & Objektivismus: & \multicolumn{3}{|c|}{$(+)$ Korrespondenz $\leftrightarrow$ Subjektivität (-) } \\
\hline & Empirismus: & (+) Empirisch & $\leftrightarrow$ & nalytisch (-) \\
\hline \multirow{2}{*}{ Ethische Ideologien } & Relativismus: & $(+)$ Kontextuell & $\leftrightarrow$ & Jniversell (-) \\
\hline & Idealismus: & (+) Mittel & $\leftrightarrow$ & Zweck (-) \\
\hline
\end{tabular}

Damit eröffnet das Modell einen siebendimensionalen Raum, in dem verschiedene kulturelle Milieus im Journalismus als Koordinaten abgebildet, systematisch verglichen und taxonomisch eingeordnet werden können. Jede der sieben Dimensionen bildet ein Kontinuum mit zwei idealtypischen Endpunkten, die allerdings in der „realen Welt“ des Journalismus in dieser Form kaum vorkommen. Das Modell lässt sich dabei von der Annahme leiten, dass sich die vorgenannten sieben Hauptdimensionen von Journalismuskultur in allen Nationen, Medienorganisationen und professionellen Journalistenmilieus manifestieren. Allerdings wird die relative Gewichtung der einzelnen Dimensionen jeweils unterschiedlich ausfallen. Diese relativen Unterschiede bringen ein Universum hervor, in dem zum Teil sehr verschiedene journalistische Kulturen ihre Identität artikulieren.

\section{Institutionelle Rollen}

Institutionelle Rollen bündeln Vorstellungen über die Funktionen und Leistungen von Journalismus in der Gesellschaft und werden in der Literatur auch als berufliche Rollenverständnisse beschrieben. Frühe Klassifikationsversuche journalistischer Selbstwahrnehmungen haben „neutrale“ von „teilnehmenden“ Rollen (Cohen 1963: 20) bzw. den „Gatekeeper“ vom „Advocate“ (Janowitz 1975: 618-9) unterschieden. Während der Gatekeeper einem Berufsverständnis folgt, das auf Werte wie Objektivität, Neutralität 
und Distanz sowie auf eine klare Trennung von Fakten und Meinung setzt, geht es beim anwaltschaftlichen Ansatz um eine aktive Teilnahme an Prozessen, die Gegenstand der Berichterstattung sind. In einem theoretischen Annäherungsversuch haben Donsbach und Patterson (2003: 298ff.) hierfür eine zweidimensionale Unterscheidung eingeführt, bei der journalistische Rollenverständnisse einerseits auf einem Kontinuum zwischen „passiv“ und „aktiv“ und andererseits zwischen „neutral“ und "anwaltschaftlich“ verortet werden können. In einer ganzen Reihe von Studien u. a. in Ägypten, Bangladesch, Brasilien, Deutschland, Indonesien, Tansania, in Nepal sowie in der Schweiz und den USA wurde versucht, die dimensionale Struktur von Rollenverständnissen faktorenanalytisch zu ermitteln (vgl. Hanitzsch 2004; Herscovitz 2004; Marr et al. 2001; Ramaprasad 2001; Ramaprasad \& Hamdy 2006; Ramaprasad \& Kelly 2003; Ramaprasad \& Rahman 2006; Scholl \& Weischenberg 1998; Weaver et al. 2006). In Weiterentwicklung dieser Ansätze soll hier ein Dimensionierungsvorschlag vorgestellt werden, der die Varianz in beruflichen Selbstverständnissen in ihrer globalen Vielfalt abbilden und dabei Komplexität hinreichend reduzieren kann. Drei Hauptdimensionen werden vorgeschlagen: Interventionismus, Machtdistanz und Marktorientierung.

\subsection{Interventionismus}

Interventionismus beschreibt das Maß, in dem Journalisten offensiv für bestimmte gesellschaftliche Werte eintreten bzw. sich in den Dienst einer Mission stellen. Diese Dimension, bei Himelboim und Limor (2005: 9) als ,involvement sequence“ bezeichnet, erstreckt sich von „passiv“ (negativer Pol) zu „Intervention“ (positiver Pol). Zwei grundsätzliche Typen von Journalisten können hier idealtypischerweise unterschieden werden: Der eine ist anwaltschaftlich, Partei ergreifend und beteiligt sich am Geschehen, der andere distanziert, unbeteiligt sowie den Werten von Objektivität und Unparteilichkeit verpflichtet. Der passive Pol dieser Dimension weist eine Nähe zu Rollenmodellen des neutralen Nachrichtenvermittlers („neutral disseminator“) und des Gatekeepers auf (vgl. Cohen 1963; Janowitz 1975; Weaver \& Wilhoit 1991: 115). Journalisten auf dieser Seite des Kontinuums lassen sich von konventionellen Werten des westlichen Professionalisierungsmodells (Objektivität, Neutralität, Fairness, Unvoreingenommenheit, Unabhängigkeit etc.) leiten, das insbesondere den Mainstream in den USA kennzeichnet.

In journalistischen Kulturen, die einem solchen Verständnis folgen, herrscht ein Rollenmodell vor, das Journalismus im gesellschaftlichen Kontext hauptsächlich eine Informationsfunktion zuschreibt. Während sich das Dogma der Neutralität und Unparteilichkeit in den meisten demokratischen Gesellschaften gemeinsam mit der institutionellen Trennung von Medien und Staat entwickelt hat, operieren Medienorganisationen in anderen Ländern keinesfalls oder nur bedingt unabhängig. Dies ist etwa der Fall in China, Singapur und Simbabwe - aber zum Teil durchaus auch innerhalb der westlichen Welt, z. B. in Italien (vgl. Golding 1977: 300; McQuail 2000: 253). In einer ganzen Reihe von Ländern (so etwa in weiten Teilen Südamerikas) ist die Transformation von einem anwaltschaftlichen politischen Journalismus hin zum Professionalisierungsmodell noch längst nicht abgeschlossen. Es ist daher anzunehmen, dass Journalisten in dieser Region stärker zu einem interventionistischen Verständnis tendieren.

Ein interventionistisches Rollenmodell wird in der Literatur mit den Begriffen des "Teilnehmers“ („participant“), „Anwalts“ („advocate“) und „Missionars“ („missionary“) charakterisiert (vgl. Cohen 1963; Donsbach \& Patterson 2003; Janowitz 1975; Köcher 1986). Journalisten nehmen hier in Bezug auf den Gegenstand der Berichterstattung eine stärker aktive und engagierte Rolle ein. Berichterstatter dieser Couleur mögen sich 
als Anwalt der gesellschaftlich Benachteiligten sehen - oder aber als Sprachrohr politischer und sozialer Gruppierungen. Hinter diesem Verständnis steht in jedem Fall die Absicht, sich offensiv in das Geschehen einzumischen, den (politischen) Prozess aktiv mitzugestalten, zu intervenieren, für eine Veränderung der aktuellen Zustände zu sorgen bzw. einem unerwünschten Veränderungsprozess entgegenzutreten. Interventionistische Reflexe dieser Art lassen sich in verschiedenen normativen Journalismuskonzepten beobachten, hierunter fallen u. a. die Reformbewegungen des Civic bzw. Public Journalism, Development Journalism und Peace Journalism.

\subsection{Machtdistanz}

Die zweite Dimension, Machtdistanz (Power Distance), beschreibt die Nähe von Journalisten zu den gesellschaftlichen Machtzentren und weist daher einige Ähnlichkeit mit Himelboim und Limors (2005: 10) „adversary sequence” auf. Der Terminus „Power Distance" wurde ursprünglich von Hofstede (1980) eingeführt, wo er eine der basalen Dimensionen kultureller Varianz bezeichnet. Machtdistanz im Journalismus schlägt sich in einer unterschiedlichen Sicht auf das Verhältnis zwischen Journalisten und gesellschaftlichen Machtzentren (z. B. der Regierung oder Wirtschaftsimperien) nieder. Es erstreckt sich zwischen einem Verständnis von Journalismus als „Gegenpol“ zur Macht (positiver Pol) und einer Haltung, die am besten mit „loyal“ (negativer Pol) zu beschreiben ist.

Das Verständnis von Journalismus als „Gegenpol“ zu den gesellschaftlichen Machtzentren wurzelt in einer westlich-liberalen Tradition, die Journalisten im demokratischen Prozess die Funktion der „vierten Gewalt“ („Fourth Estate“) im Staate zuweist. Berichterstatter, die einem solchen Verständnis folgen, sehen sich selbst in der Rolle des unnachgiebigen und unerbittlichen Wächters der Demokratie, der öffentliche Aussagen von Politikern mit einer stets skeptischen - und zuweilen sogar feindseligen - Haltung begegnet. Politische Prozesse werden einer unabhängigen und kritischen Prüfung unterworfen, Missstände vorbehaltlos aufgezeigt (vgl. Fuller 1996: 26; Gans 1979: 295; McQuail 2000: 161; Schramm 1964: 127). In seiner extremen Ausprägung kann ein solches Rollenmodell der kritischen Kontrolle in ein gegnerschaftliches Verständnis umschlagen, das in der angelsächsischen Literatur als „adversarial journalism“ beschrieben wird.

Obgleich viele Journalisten in liberalen Demokratien ein Verständnis von Journalismus als Gegenpol zur Macht offen zelebrieren, mag ein solches Rollenmodell in anderen kulturellen Kontexten nur schwer aufzuspüren sein - vor allem dann, wenn von den Inhalten der Berichterstattung auf die Rollenverständnisse der Autoren geschlossen werden soll. Während ein Selbstverständnis als Gegenpol zur Macht insbesondere in Teilen Asiens im Kontrast zu gesellschaftlichen Grundnormen wie Konsens und Konfliktvermeidung steht, operieren Journalisten in anderen Ländern unter erheblichen Einschränkungen von Presse- und Meinungsfreiheit. Dennoch ist es durchaus möglich, kritische Botschaften z. B. in China über „Doppelcodierungen“ zwischen den Zeilen zensierter Medientexte zu verstecken, die dann von einem entsprechend eingestellten Publikum, das sich die Inhalte über eine subversive Lesart aneignet, der Intention gemäß verstanden werden (vgl. Ma 2000: 29). In Indonesien hat sich zu Zeiten der Suharto-Diktatur die Praxis herausgebildet, kritische Botschaften ironisch zu verpacken, um damit die staatliche Zensur zu unterlaufen (vgl. Nasution 1996: 53).

Das andere Ende des Machtdistanz-Kontinuums bildet ein Journalismus, der sich loyal zu den gesellschaftlichen Machtzentren verhält. Journalismuskulturen dieses Typs 
können ihre Loyalität offen zum Ausdruck bringen, etwa durch „Propaganda-“ bzw. „Agitator-Journalismus“ (Pasti 2005: 99; Wu, Weaver \& Johnson 1996: 544), routinemäßige Selbstzensur oder als Sprachrohr der Regierung bzw. „der Partei“. Nicht auf den ersten Blick erkennbar ist eine niedrige Machtdistanz jedoch bei journalistischen Kulturen, die als „ideologischer Staatsapparat“ im Sinne Althussers fungieren - wie etwa die mexikanische „oficialista“ (Hallin 2000: 99) oder die als „protocol news“ beschriebene Berichterstattung in Uganda (Mwesige 2004: 87). Journalisten dieses Typs betrachten amtliche Informationen von vornherein als autoritativ und glaubwürdig und stellen die „offizielle Version“ des Geschehens nicht in Frage. Da die Legitimität der Deutungsmacht gesellschaftlicher Machteliten nicht in Zweifel gezogen wird, trägt diese Form des Journalismus - wenngleich auch nicht auf direkte und unmittelbare Weise - zum Erhalt des gesellschaftlichen Status quo bei.

\subsection{Marktorientierung}

Marktorientierung als dritte Dimension stellt den Produktionsfokus der Berichterstattung ins Zentrum der Betrachtung. Die Dimension lädt hoch in Journalismuskulturen, die sich von der Logik des Marktes leiten lassen, sie lädt niedrig in professionellen Milieus, die sich hauptsächlich in den Dienst des „öffentlichen Interesses“ stellen. Eine solche Unterscheidung - Markt- vs. Gemeinwohlorientierung - haben Marr et al. (2001: 121) in ihrer Befragung von Schweizer Journalisten angelegt. Die breite und öffentliche Diskussion über diese Frage, die z. B. immer wieder gern im Zuge von anstehenden Erhöhungen der Rundfunkgebühren thematisiert wird, zeigt die Zentralität dieser Dimension für die Unterscheidung journalistischer Kulturen. Denn die Medien können das Publikum auf zweierlei Weise ansprechen: in ihrer Rolle als Staatsbürger („citizen“) oder als Konsument - wobei die offensichtlich antagonistische Beziehung zwischen diesen beiden Extrempolen eine allmähliche Verschiebung hin zu einem Publikumsbild als Konsument durchläuft.

Journalismuskulturen, die stärker dem Gemeinwohl verpflichtet sind, sprechen das Publikum in seiner Rolle als Staatsbürger an. Es wird davon ausgegangen, dass die Hauptfunktion von Journalismus darin besteht, die Menschen mit den Informationen zu versorgen, die zu einer aufgeklärten Teilhabe an der freiheitlichen Demokratie notwendig sind (vgl. Kovach \& Rosenstiel 2001: 17). Journalismus ist nach diesem Verständnis ein Kommunikator-zentrierter Dienst an der Gesellschaft zur Gewährleistung von politischer Partizipation und einer informierten Wahlentscheidung. Dieser Pol der Dimension Marktorientierung schließt wesentliche Teile der Verantwortungsideologie im Journalismus ein, wie sie ursprünglich von der Commission on Freedom of the Press (1947: 92) artikuliert wurde: „[T]he press itself should assume the responsibility of providing the variety, quantity, and quality of information and discussion which the country needs." Gunaratne (2006: 15) weist jedoch zurecht darauf hin, dass das Konzept der sozialen Verantwortung in westlichen und östlichen Kulturen unterschiedlich verstanden wird. Wie die Debatte über „asiatische Werte“ im Journalismus gezeigt hat, wird Verantwortung in diesem Kontext mit der Wahrung sozialer Harmonie und Anerkennung von Autorität in Verbindung gebracht, wobei an die Medien die Erwartung herangetragen wird, vor allem solche Berichterstattung zu unterlassen, die eine potenzielle Gefahr für bestehende Ordnungen darstellen könnten (vgl. Masterton 1996; Xiaoge 2005). Die Idee eines am Gemeinwohl orientierten Journalismus findet sich darüber hinaus im Public bzw. Civic Journalism, der in den USA als Antwort auf die zunehmende Politikverdrossenheit der Medien und des Publikums entstanden ist (vgl. Rosen 2000: 680). 
Eine hohe Marktorientierung äußert sich in einer Fokussierung der Berichterstattung auf den Alltag sowie die alltäglich-banalen Bedürfnisse der Rezipienten als individuelle Konsumenten. Im Zentrum steht weniger das, was das Publikum wissen „sollte“ (um z. B. am politischen Geschehen zu partizipieren), sondern das, was das Publikum vermeintlich bewegt, einschließlich der persönlichen Ängste, (geheimen) Wünsche, Überzeugungen und emotionalen Erfahrungen (vgl. Campbell 2004: 217). Der Publikums- und Marktforschung wird in diesen Journalismuskulturen daher ein hoher Stellenwert beigemessen. Dabei nimmt die Perspektive des Individuums vor allem bei kommerziellen Medien an Bedeutung zu (vgl. Hallin \& Mancini 2004: 278). Die journalistische Anpassung an die Logik des Marktes manifestiert sich in einer professionellen Kultur des Service- und Ratgeber-Journalismus, der sich darauf verlegt, dem Publikum bei seinem Alltagsmanagement mit Beistand, Rat, Führung und Information zur Seite zu stehen (vgl. Eide \& Knight 1999: 526). Dies geschieht u. a. über "Consumer News" und „News-You-Can-Use“-Formate (vgl. Underwood 2001: 102). Faktorenanalytische Auswertungen von journalistischen Rollenverständnissen in Deutschland und Indonesien haben gezeigt, dass Service- und Ratgeber-Journalismus sowie Unterhaltung und Entspannung zwei Facetten ein und derselben Dimension bilden (vgl. Hanitzsch 2004: 500; Scholl \& Weischenberg 1998: 168).

\section{Epistemologien}

Die zweite Konstituente, Epistemologien, umfasst das erkenntnistheoretische Fundament von Journalismus und bildet als solches eine instrumentelle Komponente der journalistischen Praxisausübung. Die Epistemologie als Teilbereich der Philosophie beschäftigt sich allgemein mit dem Wesen der Erkenntnis, den Kriterien einer akzeptablen Beweisführung und der Geltung von Unterscheidungen zwischen „wahr" und „falsch“ (vgl. Andersen \& Baym 2004: 603). Letztlich geht es darum, was beobachtende Subjekte tatsächlich erkennen können, und ob das hierdurch generierte Wissen auch verlässlich und "wahr" ist. Für die Analyse von Journalismus ist die Betrachtung der implizit zur Geltung gebrachten Erkenntnistheorien zentral, da sich Journalisten in der Außen- und Selbstwahrnehmung der Wahrheit verpflichtet fühlen und die Legitimität von Journalismus deshalb mit dem Wahrheitsanspruch fest verknüpft ist (vgl. Ekström 2002: 260; Kovach \& Rosenstiel 2001: 37). Prinzipiell befassen sich epistemologische Überlegungen im Journalismus mit der eher praktischen Frage, ob Berichterstattung überhaupt „objektive“ und wertfreie „Wahrheiten“ über den journalistischen Gegenstandsbereich liefern kann, und welche Formen der Beweisführung als akzeptabel gelten können. Allgemein lassen sich im Hinblick auf die epistemologischen Grundlagen des Journalismus zwei relevante Hauptdimensionen identifizieren: Objektivismus und Empirismus.

\subsection{Objektivismus}

Objektivismus als erste Dimension setzt an der Frage an, ob die objektive Wahrheit der menschlichen Erkenntnis prinzipiell zugänglich ist. Dabei geht es weniger um ein prozedurales Verständnis im Sinne von Objektivität als Methode, sondern um eine philosophisch-absolute Sicht auf Objektivität als Möglichkeit. Die Unterscheidung verläuft hier idealtypisch zwischen einer Auffassung, wonach Wahrheit nichts anderes als die Übereinstimmung zwischen dem "Gesagten“ und dem „tatsächlich Existenten“ ist (Merill \& Odell 1983: 70), und einem Subjektivitäts-Verständnis, das sich darauf beruft, dass jede Nachrichtenproduktion der Selektivität unterworfen ist und dass Realitätser- 
kenntnis auf dem menschlichen Urteilsvermögen aufsetzt - und damit einem Prozess von Bewertungen unterliegt.

In Journalismuskulturen, die zur Korrespondenz-These (positiver Pol) tendieren, herrscht ein eher totalitäres Wahrheitsverständnis vor, das sich ein wesentliches Prinzip des Erkenntnisfundamentalismus (bzw. der erkenntnistheoretischen Theorie der Letztbegründung) zu eigen macht: Demnach ist Wahrheit die Übereinstimmung zwischen der „wahren“ Gestalt des tatsächlich Existenten und seinem geistigen Abbild. Darüber hinaus nährt sich das Wahrheitsverständnis dieser Journalismuskulturen aus dem philosophischen Realismus, bei dem die Objekte der Wahrnehmung als unabhängig von der Existenz eines wahrnehmenden Subjekts betrachtet werden (vgl. Andersen \& Baym 2004: 590; Dancy 1985: 144). Journalisten in diesen professionellen Milieus bekennen sich zur Möglichkeit der objektiven Erkenntnis über die spiegelbildhafte Abbildung der Realität in ihrer „wahren“ Beschaffenheit. Der Beobachter und der Gegenstand der Beobachtung bilden zwei unabhängige Kategorien, und die Wahrheitstreue der Medienrealität kann anhand ihrer Übereinstimmung mit der objektiven Realität gemessen werden. Demzufolge muss die Realität, um größtmögliche Objektivität zu gewährleisten, so genau und präzise wie möglich beschrieben werden.

Subjektivisten (negativer Pol) hingegen stellen die aktive Konstruktionsleistung des Journalisten in Rechnung. Sie lassen sich von dem konstruktivistischen Zweifel an der Existenz einer absoluten und objektiven Wahrheit leiten. Nachrichten sind demnach nur eine Repräsentation von Weltkomplexität, und wie alle Repräsentationen sind sie unweigerlich selektiv und an subjektive Interpretationen gebunden (vgl. Schudson 2003: 33). Wahrheit kann diesem Verständnis zufolge nicht von menschlicher Subjektivität und vom Kontext, in dem sie entsteht, getrennt werden, womit eine wertfreie Erkenntnis letztlich unmöglich ist. Eine solche erkenntnistheoretische Haltung lässt sich auch in Einklang mit einigen Strängen der fernöstlichen Philosophie bringen, in denen die objektive Realität und ihr phänomenales Abbild - Substanz und Form - unauflösbar miteinander verbunden sind (vgl. Cheng 1987). Dies mag vielleicht die Ablehnung, die viele Journalisten in Asien dem Dogma des objektiven Journalismus entgegenbringen, zumindest teilweise erklären.

Subjektivistische Berichterstattungsmuster wie der „Interpretative“ und „Neue“ Journalismus bringen jedoch nicht zwangsläufig eine Hinwendung zum Relativismus mit sich. Die Wahrheit, so lässt sich nach diesem Verständnis schlussfolgern, entsteht erst im Zusammenspiel einer potenziell unendlichen Zahl von subjektiven Versionen, die miteinander um die Deutungshoheit konkurrieren. Ein solches Verständnis der diskursiven Annäherung an die Wahrheit hat Oleg Poptsov, der frühere Chef des russischen Fernsehsenders RTR in die knappen, aber treffenden Worte gekleidet: „there is no such thing as objectivity. Objectivity is a sum total of subjectivities" (zitiert nach McNair 2000: 90).

\subsection{Empirismus}

Die zweite Dimension der Epistemologien-Konstituente beschäftigt sich mit der Frage, auf welche Weise Journalisten bestimmte Aussagen als „wahr" auszeichnen und ihnen damit vor dem Publikum Geltung verschaffen. Idealtypisch kann dies auf zweierlei Art geschehen: empirisch oder analytisch. In Journalismuskulturen, die hauptsächlich auf eine empirische Begründung von „Wahrheit" setzen (positiver Pol), dominiert eine Sicht des Erkenntnisfundamentalismus, wonach aller Erkenntnis die Erfahrung vorausgeht (vgl. Dancy 1985: 53). Journalisten in diesen kulturellen Milieus geben faktischem Wis- 
sen Vorrang gegenüber analytischem Apriori-Wissen (vgl. Merill \& Odell 1983: 53). Die Auszeichnung von Aussagen als „wahr“ wird geleistet über Inaugenscheinnahme, Beobachtung und (eigene) Erfahrung sowie über die Beibringung faktischer Beweise und Augenzeugen des Geschehens. Die prozeduralen und methodischen Aspekte der Berichterstattung - und hier vor allem der journalistischen Recherche und Faktenprüfung - gewinnen hierbei eine besondere Bedeutung. Im Falle des so genannten Präzisionsjournalismus geschieht dies zum Beispiel über quasisozialwissenschaftliche Methoden. In einer radikalen Ausprägung äußert sich diese Auffassung in einer journalistischen Praxis, die sich auf ein Protokollieren von Ereignissen und Tatsachen beschränkt und die Fakten „für sich selbst“ sprechen lässt.

Am negativen Pol des Empirismus-Kontinuums geschieht die Auszeichnung von Aussagen als „wahr“ vorrangig auf analytischem Wege. Hier dominieren weniger die faktischen Elemente, sondern vielmehr Ideen, Argumente, Werte, Meinung und Analyse - und damit Elemente des erkenntnistheoretischen Rationalismus und Idealismus. Journalisten in diesen professionellen Milieus weisen dem analytischen Wissen eine weitaus höhere Bedeutung zu, wobei „Wahrheit“ als eine von „Fakten“ weitgehend unabhängige Kategorie betrachtet wird (vgl. Merill \& Odell 1983: 54). Ein solcher analytischer Journalismus, der in der Regel die Form von Meinung und Kommentar annimmt, verpflichtet dabei keineswegs zu Neutralität: Die berufliche Reputation von Kommentatoren, Leitartiklern und Kolumnisten gründet sich nicht in konventionellen Werten wie Objektivität, Fairness und Ausgewogenheit, sondern in ihrer Fähigkeit zu einer stringenten und in sich geschlossenen Argumentation. Beide Extremformen - analytischer und empirischer Journalismus - sind in der journalistischen Praxis in reiner Form jedoch nur selten anzutreffen. Wie im Fall des Interpretativen Journalismus nimmt die Berichterstattung zumeist eine Position irgendwo zwischen den Endpunkten des Kontinuums ein.

Die Unterscheidung zwischen den beiden epistemologischen Dimensionen mag dabei zuweilen nicht immer klar erkennbar sein. Während der positive Endpunkt der Empirismus-Dimension eine starke Nähe zum Korrespondenz-Pol im Objektivismus aufweist, liegt die Verbindung zwischen einem analytischen und einem subjektivistisch gefärbten Journalismus unmittelbar auf der Hand. Dennoch lassen sich beide Dimensionen analytisch voneinander trennen, denn während Objektivismus verschiedene Positionen hinsichtlich der Erkennbarkeit von Realität markiert, umfasst Empirismus als theoretisches Konstrukt die relative Gewichtung von Fakten und Analyse. Auch wenn es auf den ersten Blick kontraintuitiv erscheint: Auch Kommentatoren und Leitartikler, die in ihren Beiträgen insbesondere mit analytischem Apriori-Wissen arbeiten, können sich dem positiven Pol der Objektivismus-Dimension verpflichtet fühlen. Denn bestimmte, weitgehend universell gültige Werte wie „Frieden“" und „menschliche Würde“ lassen sich so objektivieren, dass sie auch als quasiobjektive „Fakten“ behandelt werden können.

\section{Ethische Ideologien}

Ethische Ideologien als dritte Konstituente von Journalismuskulturen beziehen sich auf den Umgang mit Standards, Moral- und Wertvorstellungen, die im Beruf gelten oder angestrebt werden (vgl. Thomaß 1998: 18). Ethik ist somit als Moralphilosophie die Reflexion über Moral. Moralische Werte sind an den jeweiligen kulturellen Kontext gebunden, in den sie eingebettet sind. Sie bilden mithin einen wesentlichen Aspekt kultureller Varianz, der in der Analyse von Journalismuskulturen als Kontext verstanden 
werden sollte. Zwar gelten einige dieser Elemente als potenzielle Kandidaten für prototypische Werte einer „universellen“ Journalismusethik, die meisten von ihnen stehen allerdings in der Tradition westlicher Gesellschaftsmodelle. Diese Werte umfassen u. a. Wahrheit, Aufrichtigkeit, Vollständigkeit, Ausgewogenheit, Unparteilichkeit, Verantwortung, Unabhängigkeit, Minimierung von Schaden (durch die Berichterstattung) sowie Respekt vor der Privatsphäre (vgl. Cooper 1990: 3; Elliott 1988: 29; Herrscher 2002: 280f.; Perkins 2002: 203f.; Ward 2005: 13). In vielen nichtwestlichen Kulturen, die den Konzepten der sozialen Einheit und Konfliktvermeidung Vorrang einräumen, besitzen zumindest einige dieser Werte jedoch nur eingeschränkte Geltungskraft (vgl. Perkins 2002: 205). Verschiedene Studien kommen übereinstimmend zu dem Ergebnis, dass die ethischen Maßstäbe im Journalismus vor allem durch nationale Kontexte bestimmt werden (vgl. Berkowitz, Limor \& Singer 2004: 176; Weaver 1998: 473).

Moralische Werte taugen aufgrund ihres geringen Abstraktionsniveaus nur bedingt als Fundament zur Beschreibung der kulturellen Varianz professioneller Praktiken. Anstatt an den kulturspezifischen Inhalten einer journalistischen Berufsethik anzusetzen, erscheint es daher sinnvoller zu fragen, wie Journalisten mit Situationen umgehen, die aus ethischer Sicht potenziell problematisch erscheinen. Hier unterscheidet Keeble (2005: 54f.) vier Hauptströmungen: Der „professionelle Standard-Ansatz“ betont die Verbindlichkeit kodifizierter Berufsnormen (z. B. Pressekodex) und redaktioneller Richtlinien, wohingegen der „liberal-professionelle Ansatz“ ein relativ heterogenes Bündel von Auffassungen bildet, die sich in aktuellen Streitfragen aus unterschiedlichen Gründen vom Standard-Ansatz abgrenzen. Der „zynische Ansatz“ misst ethischen Überlegungen im Journalismus eine relativ geringe Bedeutung bei, während „ethische Relativisten“ einen Ansatz bevorzugen, der ethischen Dilemmas mit Ad-hoc-Lösungen begegnet.

Einen in theoretischer Hinsicht ambitionierten Versuch zur Klassifizierung ethischer Ansätze im Journalismus hat Plaisance (2005) unternommen, der sich von den Arbeiten des Psychologen Donelson R. Forsyth inspirieren ließ. Forsyth (1980: 175ff.; 1981: 218f.) typologisiert ethische Ideologien anhand von zwei kontinuierlichen Dimensionen: Relativismus steht dabei für die Anerkennung bzw. Nichtanerkennung der Geltung von universellen ethischen Regeln. Individuen können demnach die Universalität moralischer Werte prinzipiell zurückzuweisen (positiver Pol) oder aber ihre persönlichen Moralvorstellungen von universellen ethischen Regeln anleiten lassen (negativer Pol). Die zweite Dimension, Idealismus, bezieht sich auf die Konsequenzen des Umganges mit ethischen Dilemmas. Idealtypisch kann hier unterschieden werden zwischen zwei gegensätzlichen Auffassungen: Einerseits wird davon ausgegangen, dass die wünschenswerten Handlungsresultate immer nur mit der moralisch „richtigen“ Handlung (dem rechten Mittel) erreicht werden sollten (positiver Pol). Die weniger idealistische Auffassung stellt hingegen den Handlungszweck in den Vordergrund. Demnach kann auch eine „unmoralische“ Handlung Mittel zum Zweck sein, wobei ein verhältnismäßiger Schaden hingenommen werden kann, solange er zweckdienlich erscheint (negativer Pol). In dieser Dimension findet sich daher die traditionelle Unterscheidung in eine deontologische und eine teleologische Ethik wieder.

Eine Kreuzung dieser beiden Dimensionen führt zu einer Typologie von vier unterschiedlichen „ethischen Ideologien“3 (vgl. Abb. 2). Absolutisten sind davon überzeugt, dass wünschenswerte Handlungsresultate immer nur mit dem moralisch richtigen Mittel

3 Forsyth selbst liefert keinen Hinweis darauf, wo er seine Vorstellung von „Ideologie” konkret verortet. Durch die Art und Weise seiner Verwendung ist jedoch klar, dass er Ideologie als ein spezifisches Bündel von Werten und Einstellungen versteht. 
erreicht werden können, wobei universell geltende Normen den Rahmen für mögliches Handeln vorgeben. Situationisten sind zwar ebenfalls idealistisch, sie neigen jedoch zu der Auffassung, dass universelle Moral nur eine begrenzte Geltungskraft besitzt, da jede spezifische Situation jeweils ihre individuelle Lösung erfordert. Subjektivisten lassen ihre Handlungen wie Situationisten von persönlichen Einschätzungen leiten, sie stellen dabei jedoch in Rechnung, dass der Zweck auch unmoralische Mittel rechtfertigen kann. Exzeptionisten wiederum erkennen die Geltung universeller ethischer Regeln an, sind jedoch gleichzeitig offen für Ausnahmen von dieser Regel, sofern sich damit gewünschte Handlungsresultate erzielen bzw. negative Folgen abwenden lassen.

Diese vier ethischen Ideologien finden sich durchaus auch in der Journalismusliteratur wieder. Der situationistische Ansatz ist Gegenstand einer breiten Debatte (vgl. u. a. Merill \& Odell 1983: 94) und ähnelt dem ethischen Relativismus bei Keeble (2005). Journalistische Situationisten sind davon überzeugt, dass ethische Entscheidungen nicht in der abstrakten Vorstellung getroffen werden können, sondern jeweils den Bedingungen der spezifischen Situation unterliegen. Der Absolutismus wiederum trägt Züge von Sanders' (2003: 30-1) „deontologischem“ bzw. Keebles professionellem StandardAnsatz, wohingegen Subjektivisten Grundzüge des zynischen Ansatzes in sich tragen. Angewendet auf die Bewertung unkonventioneller und potenziell problematischer Recherchemethoden (z. B. Verschwiegenheitszusagen nicht einhalten, Druck auf Informanten ausüben, unautorisiert private Dokumente verwenden) würden situationistische Journalisten ihr abschließendes Urteil von der konkreten Situation abhängig machen. Subjektivisten würden unkonventionelle Recherchemethoden prinzipiell gutheißen, solange sie dazu beitragen, die gewünschten Handlungsresultate zu erzielen (z. B. um bestimmte Informationen zu erhalten). Absolutistische Journalisten würden problematische Recherchemethoden jedoch in jeden Fall zurückweisen, zumindest so lange, wie sie im Widerspruch zum professionellen Verhaltenskodex und zu redaktionellen Grundregeln stehen. Exzeptionisten würden sich dieser Haltung im Grundsatz anschließen, sie könnten sich den Einsatz unkonventioneller Praktiken im Ausnahmefall allerdings vorstellen.

Abbildung 2: Klassifikation von ethischen Ideologien nach Forsyth (1980: 176)

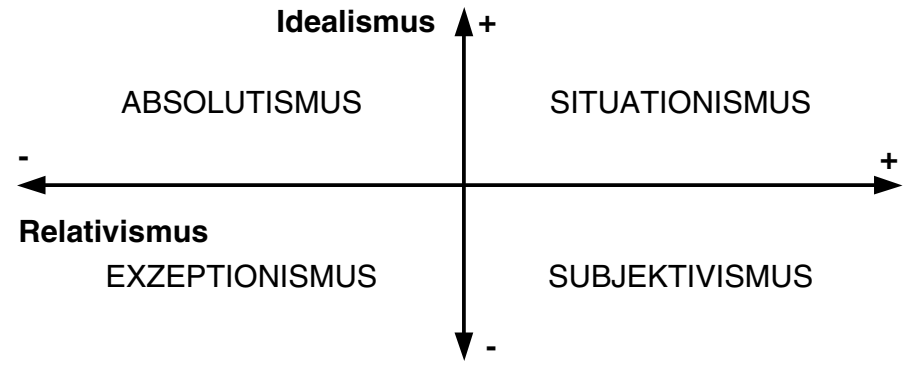

\section{Einordnung und Ausblick}

Die hier vorgeschlagene Modellierung von Journalismuskultur führt verschiedene und oftmals weitgehend isolierte Diskurse zusammen. Denn journalistische Kulturen - wie der objektive Journalismus oder Public Journalism - werden zumeist im Hinblick auf die gesellschaftliche Funktion von Journalismus, die berufsethischen Defizite oder (be- 
deutend seltener) epistemologische Fragen diskutiert, ohne diese drei wesentlichen kulturellen Facetten von Journalismus miteinander in Beziehung zu setzen. Demgegenüber bietet der hier vertretene Ansatz ein theoretisches Raster, um verschiedene Journalismuskulturen (neutral-objektiver Journalismus, Service-Journalismus, anwaltschaftlicher Journalismus, Development Journalism etc.) in einem universellen Koordinatensystem empirisch zu verorten. Das Modell erlaubt somit eine systematische „Kartografie“ und Typologisierung professioneller Orientierungen - sowie ihren Vergleich - in einem siebendimensionalen Raum von Journalismuskultur.

Im nächsten Schritt wird das theoretische Konstrukt in ein empirisches Forschungsinstrument übersetzt. Dies geschieht zurzeit im Rahmen des multinationalen „Worldsof-Journalisms“-Projekts, an dem Forscher aus 14 Ländern beteiligt sind. Die Auswahl der Länder folgt dem Most Different Systems Design (vgl. Przeworski \& Teune 1970: $31 \mathrm{ff}$. ), d. h., es wurde auf eine größtmögliche kulturelle Heterogenität geachtet. Die sieben Dimensionen werden jeweils über mehrere Items operationalisiert, immer zwei bis drei Items für den positiven und den negativen Pol einer jeden Dimension. Im Herbst 2007 werden in jedem Land insgesamt 100 Journalisten aus 20 Medienbetrieben telefonisch befragt, ein Quotierungsverfahren sorgt dabei für die größtmögliche Vergleichbarkeit der Stichproben. Die Güte des Modells wird für alle Länder separat über eine Analyse der Faktorladungsmuster mit konfirmatorischen Faktorenanalysen sowie über Faktorstrukturvergleiche und Faktorkongruenzkoeffizienten geprüft (vgl. Bortz 1999). Das Modell kann dann als tauglich eingestuft werden, wenn sich die theoretisch angenommene Struktur in allen Ländern empirisch reproduzieren lässt.

Gleichzeitig mit der Befragung werden Daten über die Redaktionen und Medienorganisationen, in denen die Journalisten beschäftigt sind, sowie die nationalen Mediensysteme erhoben bzw. zusammengetragen. Damit liegen Daten auf insgesamt drei Analyseebenen vor: der Ebene der Journalisten, Organisationen und Mediensysteme. Über das komplexe Verfahren der Mehrebenenanalyse (Multilevel Modeling; vgl. Ditton 1998; Langer 2004) werden dann jene Einflussfaktoren identifiziert, die hauptsächlich zur Varianz von Journalismuskulturen beitragen. Mittelfristig soll das hier entwickelte theoretische und methodische Inventar Forschungsinstrumente ersetzen, die trotz ihrer breiten Verwendung nicht für den Kulturvergleich entwickelt wurden. Dies gilt insbesondere für die Skalen zur Messung von professionellen Selbstverständnissen und berufsethischen Einstellungen, die von Weaver und Wilhoit (1991) kultiviert wurden.

\section{Literatur}

Anderson, James A. \& Geoffrey Baym (2004): Philosophies and Philosophic Issues in Communication, 1995-2004. In: Journal of Communication 54 (4): 589-615.

Archer, Margaret S. (1996): Culture and Agency: The Place of Culture in Social Theory. Revised Edition. Cambridge: Cambridge University Press.

Berkowitz, Dan, Yehiel Limor \& Jane Singer (2004): A Cross-Cultural Look at Serving the Public Interest: American and Israeli Journalists Consider Ethical Scenarios. In: Journalism 5 (2): 159-181.

Blumler, Jay G. (ed.) (1983): Communicating to Voters: Television in the First European Parliamentary Elections. London \& Beverly Hills, CA: Sage.

Bortz, Jürgen (1999): Statistik für Sozialwissenschaftler. 5., vollst. überarb. Aufl. Berlin u. a.: Springer.

Campbell, Vincent (2004): Information Age Journalism: Journalism in an International Context. London: Arnold.

Cheng, Chung-Ying (1987): Chinese Philosophy and Contemporary Human Communication 
Theory. In: D. Lawrence Kincaid (ed.): Communication Theory: Eastern and Western Perspectives. San Diego, CA: Academic Press, 23-43.

Cohen, Bernhard Cecil (1963): The Press and Foreign Policy. Princeton, NJ: Princeton University Press.

Commission on Freedom of the Press (1947): A Free and Responsible Press. A General Report on Mass Communication: Newspapers, Radio, Motion Pictures, Magazines, and Books. Chicago: University of Chicago Press.

Cooper, Tom (1990): Comparative International Media Ethics. In: Journal of Mass Media Ethics 5 (1): 3-14.

Dancy, Jonathan (1985): An Introduction to Contemporary Epistemology. Malden, MA, Oxford \& Victoria: Blackwell.

Deuze, Mark (2002): National News Cultures: A Comparison of Dutch, German, British, Australian and U.S. Journalists. In: Journalism \& Mass Communication Quarterly 79 (1): 134-149.

Deuze, Mark (2005): What Is Journalism? Professional Identity and Ideology of Journalists Reconsidered. In: Journalism 6 (4): 442-464.

Ditton, Hartmut (1998): Mehrebenenanalyse. Grundlagen und Anwendungen des Hierarchisch Linearen Modells. Weinheim: Juventa.

Donsbach, Wolfgang (1995): Lapdogs, Watchdogs and Junkjard Dogs. In: Media Studies Journal 9 (4): 17-30.

Donsbach, Wolfgang \& Thomas Patterson (2003): Journalisten in der politischen Kommunikation: Professionelle Orientierungen von Nachrichtenredakteuren im internationalen Vergleich. In: Frank Esser \& Barbara Pfetsch (Hrsg.): Politische Kommunikation im internationalen Vergleich. Grundlagen, Anwendungen, Perspektiven. Wiesbaden: Westdeutscher Verlag, 281-304.

Eide, Martin \& Graham Knight (1999): Public/Private Service: Service Journalism and the Problems of Everyday Life. In: European Journal of Communication 14 (4): 525-547.

Ekström, Mats (2002): Epistemologies of TV Journalism: A Theoretical Framework. In: Journalism 3 (3): 259-282.

Elliott, Deni (1988): All Is Not Relative: Essential Shared Values and the Press. In: Journal of Mass Media Ethics 3 (1): 28-32.

Esser, Frank (1998): Editorial Structures and Work Principles in British and German Newsrooms. In: European Journal of Communication 13 (3): 375-405.

Esser, Frank (2004): Journalismus vergleichen. Komparative Forschung und Theoriebildung. In: Martin Löffelholz (Hrsg.): Theorien des Journalismus. Ein diskursives Handbuch. Wiesbaden: VS Verlag, 151-179.

Fawcett, Liz (2002): Why Peace Journalism Isn't News. In: Journalism Studies 3 (2): 213-223.

Forsyth, Donelson R. (1980): A Taxonomy of Ethical Ideologies. In: Journal of Personality and Social Psychology 39 (1): 175-184.

Forsyth, Donelson R. (1981): Moral Judgment: The Influence of Ethical Ideology. In: Personality and Social Psychology Bulletin 7 (2): 218-223.

Fuller, Jack (1996): News Values: Ideas for an information age. Chicago \& London: University of Chicago Press.

Gade, Peter J. (2004): Newspapers and Organizational Development: Mangement and Journalist Perception of Newsroom Cultural Change. In: Journalism and Communication Monographs $6(1)$.

Gans, Herbert J. (1979): Deciding What's News: A Study of CBS Evening News, NBC Nightly News, Newsweek, and Time. New York: Pantheon Books.

Golding, Peter (1977): Media Professionalism in the Third World: The Transfer of an Ideology. In: James Curran \& Michael Gurevitch (ed.): Mass Communication and Society. London: Arnold, 291-308.

Gunaratne, Shelton A. (2006): Democracy, Journalism and Systems: Perspectives from East and West. In: Hao Xiaoming \& Sunanda K. Datta-Ray (eds.): Issues and Challenges in Asian Journalism. Singapore: Marshall Cavendish, 1-24.

Hallin, Daniel C. (2000): Media, Political Power, and Democratization in Mexico. In: James Curran \& Myung-Jin Park (eds.): De-Westernizing Media Studies. London: Routledge, 97-110. 
Hallin, Daniel C. \& Paolo Mancini (2004): Comparing Media Systems: Three Models of Media and Politics. New York: Cambridge University Press.

Hanitzsch, Thomas (2004): Journalismus in Indonesien. Akteure, Strukturen, Orientierungshorizonte. Journalismuskulturen. Wiesbaden: DUV.

Hanitzsch, Thomas (2006): Mapping Journalism Culture: A theoretical taxonomy and case studies from Indonesia. In: Asian Journal of Communication 16 (2): 169-186.

Harrison, Jackie (2000): Terrestrial Television News in Britain: The Culture of Production. Manchester: Manchester University Press.

Herrscher, Roberto (2002): A Universal Code of Journalism Ethics: Problems, Limitations, and Proposals. In: Journal of Mass Media Ethics 17 (4): 277-289.

Herscovitz, Heloiza G. (2004): Brazilian Journalists' Perceptions of Media Roles, Ethics and Foreign Influences on Brazilian Journalism. In: Journalism Studies 5 (1): 71-86.

Himelboim, Itai \& Yhiel Limor (2005): The Journalistic Societal Role: An International Comparative Study of 242 Codes of Ethics. Paper vorgestellt auf der 55. Jahrestagung der International Communication Association, New York, 26.-30. Mai 2005.

Hofstede, Geert (1980): Culture's Consequences: International Differences in Work-related Values. Beverley Hills, CA: Sage.

Hofstede, Geert (2001): Culture's Consequences. Second Edition: Comparing Values, Behaviors, Institutions and Organizations Across Nations. Thousand Oaks: Sage.

Hollifield, Ann C., Gerald M. Kosicki \& Lee B. Becker (2001): Organizational vs. Professional Culture in the Newsroom: Television News Directors' and Newspaper Editors' Hiring Decisions. In: Journal of Broadcasting \& Electronic Media, 45 (1): 92-117.

Janowitz, Morris (1975): Professional Models in Journalism: The Gatekeeper and the Advocate. In: Journalism Quarterly 52 (4): 618-626, 662.

Josephi, Beate (2006): Journalism in the Global Age: Between Normative and Empirical. In: Gazette 67 (6): 575-590.

Karmasin, Matthias (2000): Cultural Theory als kulturwissenschaftlicher Ansatz. In: Thomas Düllo, Arno Meteling, André Suhr \& Carsten Winter (Hrsg.): Kursbuch Kulturwissenschaft. Münster: Lit, 245-257.

Keeble, Richard (2005): Journalism Ethics: Towards an Orwellian Critique? In: Stuart Allan (ed.): Journalism: Critical Issues. Maidenhead: Open University Press, 54-66.

Knott, Diana L., Virginia Carroll \& Philip Meyer (2002): Social Responsibility Wins When CEO Has Been Editor. In: Newspaper Research Journal 23 (1): 25-37.

Köcher, Renate (1986): Bloodhounds or Missionaries: Role Definitions of German and British Journalists. In: European Journal of Communication 1 (1): 43-64.

Kopper, Gerd G. (2003): Journalistische Kultur in Deutschland: Bestimmung kultureller Grundmuster des Journalismus in Deutschland. In: G. G. Kopper \& P. Mancini (Hrsg.): Kulturen des Journalismus und politische Systeme. Probleme internationaler Vergleichbarkeit des Journalismus in Europa - verbunden mit Fallstudien zu Großbritannien, Frankreich, Italien und Deutschland. Berlin, 109-130.

Kovach, Bill \& Tom Rosenstiel (2001): The Elements of Journalism. London: Atlantic Books.

Kramer, Dieter (2000): Empirische und theoretische Orientierung in der Kulturwissenschaft. In: Thomas Düllo, Arno Meteling, André Suhr \& Carsten Winter (Hrsg.): Kursbuch Kulturwissenschaft. Münster: Lit, 61-72.

Langer, Wolfgang (2004): Mehrebenenanalyse. Eine Einführung für Forschung und Praxis. Wiesbaden: VS Verlag.

Loo, Eric (1996): Value Formation in Journalism Education in Asia. In: Murray Masterton (ed.): Asian Values in Journalism. Singapore: AMIC, 114-123.

Lünenborg, Margreth (2005): Journalismus als kultureller Prozess. Zur Bedeutung von Journalismus in der Mediengesellschaft. Ein Entwurf. Wiesbaden: VS Verlag.

Ma, Eric Kit-wai (2000): Rethinking media studies. The case of China. In: James Curran \& MyungJin Park (eds.): De-Westernizing Media Studies. London: Routledge, 21-34.

Machill, Marcel (Hrsg.) (1997): Journalistische Kultur. Rahmenbedingungen im internationalen Vergleich. Opladen: Westdeutscher Verlag. 
Marr, Mirko, Vinzenz Wyss, Roger Blum \& Heinz Bonfadelli (2001): Journalisten in der Schweiz. Eigenschaften, Einstellungen, Einflüsse. Konstanz: UVK.

Massey, Brian L. \& Li-jing Arthur Chang (2002): Locating Asian Values in Asian Journalism: A Content Analysis of Web Newspapers. In: Journal of Communication 25 (4): 987-1003.

Masterton, Murray (ed.) (1996): Asian Values in Journalism. Singapore: AMIC.

McNair, Brian (2000): Journalism and Democracy: An Evaluation of the Political Public Sphere. London \& New York: Routledge.

McQuail, Denis (2000): McQuail's Mass Communication Theory. London, Thousand Oaks, CA $\&$ New Delhi: Sage.

Merill, John C. \& Jack S. Odell (1983): Philosophy and Journalism. New York: Longman.

Mwesige, Peter G. (2004): Disseminators, Advocates and Watchdogs: A Profile of Ugandan Journalists in the New Millennium. In: Journalism 5 (1): 69-96.

Nasution, Zulkarimein (1996): Social and Cultural Influences on Journalism Values in Asia. In: Murray Masterton (ed.): Asian Values in Journalism. Singapore: AMIC, 52-55.

Pasti, Svetlana (2005): Two Generations of Contemporary Russian Journalists. In: European Journal of Communication 20 (1): 89-115.

Perkins, Michael (2002): International Law and the Search for Universal Principles in Journalism Ethics. In: Journal of Mass Media Ethics 17 (3): 193-208.

Pfetsch, Barbara (2003): Politische Kommunikationskultur. Politische Sprecher und Journalisten in der Bundesrepublik und den USA im Vergleich. Wiesbaden: VS Verlag.

Pike, Kenneth (1967): Language in Relation to a Unified Theory of the Structure of Human Behavior. Second edition. The Hague: Mouton.

Plaisance, Patrick Lee (2005): An Assessment of Media Ethics Education: Course Content and the Values and Ethical Ideologies of Media Ethics Students. Paper vorgestellt auf der 55. Jahrestagung der International Communication Association, New York, 26.-30. Mai 2005.

Przeworski, Adam \& Henry Teune (1970): The Logic of Comparative Inquiry. New York: Wiley.

Ramaprasad, Jyotika (2001): A Profile of Journalists in Post-Independence Tanzania. In: Gazette, 63 (6): 539-556.

Ramaprasad, Jyotika \& Naila Nabil Hamdy (2006): Functions of Egyptian Journalists: Perceived Importance and Actual Performance. In: The International Communication Gazette 68 (2): $167-185$.

Ramaprasad, Jyotika \& James D. Kelly (2003): Reporting the News from the World's Rooftop: A Survey of Nepalese Journalists. In: Gazette, 65 (3): 291-315.

Ramaprasad, Jyotika \& Shafiqur Rahman (2006): Tradition with a Twist: A Survey of Bangladeshi Journalists. In: The International Communication Gazette 68 (2): 148-165.

Reckwitz, Andreas (1997): Kulturtheorie, Systemtheorie und das sozialtheoretische Muster der Innen-Außen-Differenz. In: Zeitschrift für Soziologie 26 (5): 317-336.

Reese, Stephen D. (2001): Understanding the Global Journalist: A Hierarchy-of-Influences Approach. In: Journalism Studies 2 (2): 173-187.

Rosen, Jay (2000): Questions and Answers About Public Journalism. In: Journalism Studies 1 (4): 679-683.

Sanders, Karen (2003): Ethics \& Journalism. London: Sage.

Scholl, Armin \& Siegfried Weischenberg (1998): Journalismus in der Gesellschaft. Theorie, Methodologie und Empirie. Opladen: Westdeutscher Verlag.

Schramm, Wilbur (1964): Mass Media and National Development: The Role of Information in Developing Countries. Stanford, CA: Stanford University Press.

Schudson, Michael (2003): The Sociology of News. New York: W. W. Norton.

Shoemaker, Pamela J. \& Akiba A. Cohen (2006): News Around the World: Content, Practitioners, and the Public. New York u. a.: Routledge.

Shoemaker, Pamela J. \& Stephen D. Reese (1996): Mediating the Message: Theories of Influence on Mass Media Content. Second Edition. White Plains, NY: Longman.

Splichal, Slavko \& Colin Sparks (1994): Journalists for the 21st Century: Tendencies of Professionalization Among First-Year Students in 22 Countries. Norwood, NJ: Ablex. 
Thomaß, Barbara (1998): Journalistische Ethik: Ein Vergleich der Diskurse in Frankreich, Großbritannien und Deutschland. Opladen: Westdeutscher Verlag.

Underwood, Doug (2001): Reporting and the Push for Market-Oriented Journalism: Media Organizations as Business. In: W. Lance Bennett \& Robert M. Entman (eds.): Mediated Politics: Communication in the Future of Democracy. Cambridge: Cambridge University Press, 99-116.

Van de Vijver, Fons J. R. \& Kwok Leung (1997): Methods and Data Analysis for Cross-Cultural Research. Thousand Oaks, CA: Sage.

Ward, Stephen J. A. (2005): Philosophical Foundations for Global Journalism Ethics. In: Journal of Mass Media Ethics 20 (1): 3-21.

Weaver, David H. \& G. Cleveland Wilhoit (1991): The American Journalist. Second Edition. Bloomington, IN: Indiana University Press.

Weaver, David (1998): Journalist Around the World: Commonalities and Differences. In: David H. Weaver (ed.): The Global Journalist: News People Around the World. Cresskill, NJ: Hampton, 455-480.

Weaver, David H., Randall A. Beam, B. J. Brownley, Paul Voakes \& G. Cleveland Wilhoit (2006): The American Journalist in the 21st Century: U.S. news people at the dawn of a new millennium. Mahwah, NJ: Lawrence Erlbaum.

Weischenberg, Siegfried, Sabine von Bassewitz \& Armin Scholl (1989): Konstellationen der Aussagenentstehung. Zur Handlungs- und Wirkungsrelevanz journalistischer Kommunikationsabsichten. In: M. Kaase \& W. Schulz (Hrsg.): Massenkommunikation. Theorien, Methoden, Befunde. Opladen: Westdeutscher Verlag, 280-300.

Williams, Raymond (1958): Culture and Society, 1780-1950. London and New York: Columbia University Press.

Wu, Wei, David Weaver \& Owen V. Johnson (1996): Professional Roles of Russian and U.S. Journalists: A Comparative Study. In: Journalism \& Mass Communication Quarterly 73 (3): 534548.

Xiaoge, Xu (2005): Demystifying Asian Values in Journalism. Singapore: Marshall Cavendish.

Zelizer, Barbie (2005): The culture of journalism. In: James Curran \& Michael Gurevitch (eds.): Mass Media and Society, 4th ed. London: Hodder Education, 198-214. 